should be convenient for young people to come as well as the location should be strategic.

Disclosure No significant relationships.

\section{P018 PAY-IT-FORWARD GONORRHEA AND CHLAMYDIA TESTING AMONG CHINESE MEN WHO HAVE SEX WITH MEN: A CLUSTER RANDOMIZED CONTROLLED TRIAL}

\begin{abstract}
${ }^{1}$ Tiange Zhang*, ${ }^{2}$ Fan Yang, ${ }^{3}$ Weiming Tang, ${ }^{3}$ Wenting Huang, ${ }^{1}$ Yehua Wang, ${ }^{4}$ Marcus Alexander, ${ }^{4}$ Laura Forastiere, ${ }^{4}$ Navin Kumar, ${ }^{3}$ Katherine Li, ${ }^{5}$ Fei Zou, ${ }^{6}$ Ligang Yang, ${ }^{7}$ Guodong Mi, ${ }^{3}$ Amy Lee, ${ }^{8}$ Weizan Zhu, ${ }^{9}$ Peter Vickerman, ${ }^{3}$ Dan $W u,{ }^{6}$ Bin Yang, ${ }^{4}$ Nicholas Christakis, ${ }^{3}$ Joseph Tucker. 'University of North Carolina Project-China, Guangzhou, China; ${ }^{2}$ University of North Carolina Project - China, Guangzhou, China; ${ }^{3}$ UNC Project-China, Guangzhou, China; ${ }^{4}$ Yale Institute for Network Science, New Haven, USA; ${ }^{5}$ University of North Carolina at Chapel Hill, Department of Biostatistics, Chapel Hill, USA; ${ }^{6}$ Dermatology Hospital, Southern Medical University, Guangzhou, China; ${ }^{7}$ BlueD, Beijing, China; ${ }^{8}$ Zhitong Guangzhou LGBT Center, Guangzhou, China; ${ }^{9}$ University of Bristol, School of Social and Community Medicine, Bristol, UK
\end{abstract}

\subsection{6/sextrans-2019-sti.227}

Background Gonorrhea and chlamydia testing is poor among Chinese MSM with HIV risk. Furthermore, gonorrhea and chlamydia testing programs are poorly funded and unlinked to HIV testing services. Pay-it-forward offers an individual a gift (e.g. an STD test) and then asks whether they would like to give a gift to another person. This study assesses the effectiveness of a pay-it-forward program in increasing gonorrhea/chlamydia testing among MSM compared to standard of care.

Methods We conducted a cluster randomized controlled trial at three HIV testing sites run by MSM community-based organizations in Beijing and Guangzhou (NCT03741725). We included MSM aged 16 or older who had met indications for testing based on US CDC guidelines. Twenty clusters of 10 men each were randomized into pay-it-forward and standard of care arms. In pay-it-forward, men were offered free testing and then given the option to donate money toward future participants' tests; in standard of care, testing was offered at the standard price of $150 \mathrm{RMB}$ (US\$22). The primary outcome was dual gonorrhea/chlamydia test uptake ascertained by administrative record. Generalized estimating equations were used to assess the population-averaged effect of the pay-it-forward intervention. This analysis focuses on the primary comparison between pay-it-forward and standard of care.

Results Overall, 201 participants were recruited. Most participants were younger than 30 years old $(67.5 \%, 127 / 188)$ and had college or above education (85.1\%, 165/194). 43.1\% (69/ $160)$ reported condomless anal sex in the last three months. Gonorrhea/chlamydia testing rates were $56.4 \%(57 / 101)$ in pay-it-forward and $18.0 \%(18 / 100)$ in standard of care. Gonorrhea/chlamydia testing rates were three times higher in payit-forward compared to standard of care (RR:3.14, p $<0.001$, $95 \% \mathrm{CI}=1.80-5.45)$. $94.7 \%(54 / 57)$ of pay-it-forward participants donated toward future participants, with an average amount of 53.6RMB (US\$8).

Conclusion Findings suggest that pay-it-forward increases gonorrhea/chlamydia testing and may inform the integration of STD and HIV testing services among MSM.

Disclosure No significant relationships.

\section{P019 \\ IMPLEMENTING HOME-SAMPLING STRATEGIES TO ENGAGE YOUNG HETEROSEXUALS IN CHLAMYDIA TRACHOMATIS RE-TESTING AND PEER-TESTING}

Nicole Dukers-Muijrers*, Jeanine Leenen, Christian Hoebe. Public Health Service South Limburg, Maastricht University Medical Center (MUMC), Sexual Health, Infectious Diseases and Environmental Health, Medical Microbiology, Care and Public Health Research Institute (CAPHRI), Heerlen, Netherlands

\subsection{6/sextrans-2019-sti.228}

Background Home-sampling is an increasingly used method to promote testing for Chlamydia trachomatis. Here, we implement and evaluate home-sampling for re-testing and peer-testing in young $(<25$ years) heterosexual men and women.

Methods At our STI clinics (Limburg, Netherlands, 20152018), indexes, i.e. young heterosexuals diagnosed with chlamydia, were (a) offered a self-sampling test for a peer, and (b) offered to receive an SMS-reminder after 5 months to order a re-test for themselves and a peer. Test offers were free-of-charge self-sampling tests for chlamydia and gonorrhea (urine sample for men; vaginal and rectal swabs for women). We interviewed care providers on implementation-barriers and facilitators and performed a process evaluation on test-uptake and overall (genital or rectal) STI positivity.

Results Of 1709 indexes (1221 women): (a) 571 (33.4\%) were given a test for a peer; 211 (37.0\%) peers tested and peer-positivity was $18.5 \%$ (17.9\% in women-including 3 cases of gonorrhea and $12.8 \%$ rectal chlamydia; $19.5 \%$ in men). (b) $1048(61.3 \%)$ accepted to receive a re-test reminder; 417 $(39.8 \%)$ ordered the re-test and $266(63.8 \%)$ re-tested with $13.9 \%$ re-test positivity (15.6\% in women -including $10.4 \%$ rectal chlamydia-, $8.2 \%$ in men - including one gonorrhea case). $155 / 417(37.2 \%)$ also ordered a peer-test; $62(40 \%)$ peers tested but only $6.5 \%(n=4)$ were positive. In (a) and (b), nearly half of positive peers never tested before; rectal infections in women were frequently without a genital infection. Interviews with care-providers revealed problems in implementing logistics which could be solved by an easy-touse online system.

Conclusion A future implementation using an online system needs to improve test-kit returns by including reminders or provisionary payment, and strategies to help indexes find and motivate high risk peers. High STI positivity in peers (many first time testers) from clinic-indexes reveals the need to especially target these peers to tap into a hidden (untested) infected population.

Disclosure No significant relationships.

\section{P020 URISPONGETM FACILITATE URINE COLLECTION AND TRANSPORTATION FOR THE DETECTION OF STD WITH THE ANYPLEXTM II STI-7 ASSAY}

${ }^{1}$ Santina Castriciano*, ${ }^{2}$ Anna Archenti, ${ }^{2}$ Patrizia Biagiola, ${ }^{2}$ Debora Pasquali, ${ }^{2}$ Marina Foti. ${ }^{1}$ Copan Italia Spa, Scientific Affairs, Hamilton, Canada; ${ }^{2}$ 2ATS Città Metropolitana di Milano-Laboratorio di Prevenzione, Milano, Italy

\subsection{6/sextrans-2019-sti.229}

Background Molecular urine collection devices are not supporting bacteria culture. Copan UriSponge ${ }^{\mathrm{TM}}$ consists of a leak-proof screw-cap tube with 3 sponges containing preservative salts attached to a plastic stick to absorb and retain urine during transport and prevent bacterial overgrowth. Urines collected in UriSponge ${ }^{\mathrm{TM}}$ were compared to urine samples 